\title{
Polarimetry of binary systems: polars, magnetic CVs, XRBs
}

\author{
Tariq Shahbaz
}

\begin{abstract}
Polarimetry provides key physical information on the properties of interacting binary systems, sometimes difficult to obtain by any other type of observation. Indeed, radiation processes such as scattering by free electrons in the hot plasma above accretion discs, cyclotron emission by mildly relativistic electrons in the accretion shocks on the surface of highly magnetic white dwarfs and the optically thin synchrotron emission from jets can be observed. In this review, I will illustrate how optical/near-infrared polarimetry allows one to estimate magnetic field strengths and map the accretion zones in magnetic Cataclysmic Variables as well as determine the location and nature of jets and ejection events in X-ray binaries.
\end{abstract}

\section{Cataclysmic variables}

Cataclysmic variables (CVs) are a class of binary system in which a main sequence secondary star transfers matter to its white dwarf (WD) companion, via Roche lobe overflow. It is estimated that $\sim 20 \%$ of all CVs host a magnetic WD (mCVs) [1, 2]. In the "normal" accreting mode known as the "high-state" of mCVs, the accreting material leaving the inner Lagrangian L1 point attaches itself to the magnetic field lines of the WD close and is channelled towards its surface. However, most mCVs also show erratic and abrupt "low-states," during which accretion through the L1 point temporarily stops, presumably due to changes in the mass-transfer rate in the system, related to the atmospheric magnetic activity of the secondary star [3]. During the high-state, as the flow is collimated by the magnetic field, electrons spiral down the magnetic field lines, cooling the plasma flow by cyclotron emission. This radiation is detected as broad overlapping cyclotron humps in the optical

Tariq Shahbaz

Instituto de Astrofísica de Canarias (IAC), E-38200 La Laguna, Tenerife, Spain and Departamento de Astrofísica, Universidad de La Laguna (ULL), E-38206 La Laguna, Tenerife, Spain, e-mail: tsheiac.es 
and infrared (IR) spectrum and allows to estimate magnetic field strengths within the accretion region. Hard X-rays resulting from a post-shock region just above the accreting pole and softer ultraviolet (UV) and X-rays are emitted from a hot photospheric polar cap. During the low-states, Zeeman spectroscopy can be used to constrain the temperature and magnetic field of the WD (see Fig. 1] for a review see [4]).

Fig. 1 A schematic of the surface of the WD, showing the region of the main emission mechanisms [5].

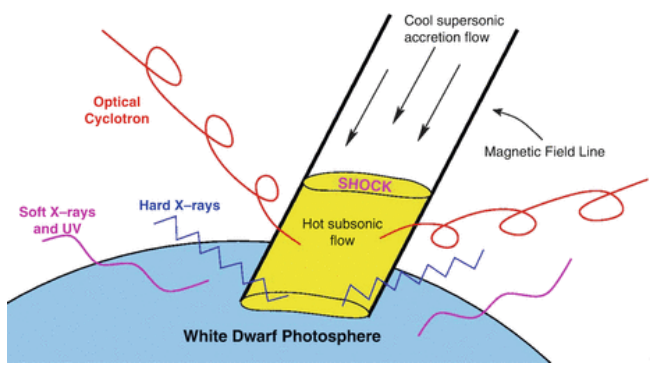

MCVs are characterized by strong X-ray emission, high-excitation optical spectra, very stable X-ray and optical periods in their light curves. They are broadly classified into two categories based upon their periodicities, which are in turn assumed to imply different magnetic field strengths. The polars (or AM Her type systems after the prototype system) contain synchronously rotating WDs, whilst in intermediate polars (IPs or DQ Her type systems) the WDs rotate asynchronously with respect to the binary orbit (see Fig. 2).

- In polars, the magnetic field strength of the WD $(>10 \mathrm{MG})$ is so strong that the gas leaving the inner Lagrangian point L1 following a ballistic trajectory, eventually attaches to the magnetic field of the WD, preventing the formation of an accretion disc as in CVs. Accretion can occur on one or both poles, depending on the orientation of the magnetic dipole of the WD. The material is fully ionized producing highly polarized cyclotron emission due to the presence of the strong magnetic field. Since a large fraction of the optical flux in polars comes from such regions, they have large polarization values. The radiation is circularly polarised when observed parallel to the magnetic field lines and linearly when observed perpendicular to the field lines. Consequently, polarimetry is a fundamental tool for classifying an object a polar. The magnetic field of the WD extends to the secondary synchronizes the spin and orbital period. Typically the polars are found to have an orbital and spin period of less than two hours (for a review see [4]).

- The IPs are asynchronous systems which have a magnetic field strength of $<10$ MG. The lower magnetic field strength compared to polars implies that matter falls inward towards the WD in much the same way as in non-magnetic CVs. However, the magnetosphere disrupts the inner portion of the accretion disc and the flow then follows the field lines in a curtain-like structure [7]. Due to the disc symmetry, the accretion curtains are present for both magnetic poles, resulting in 

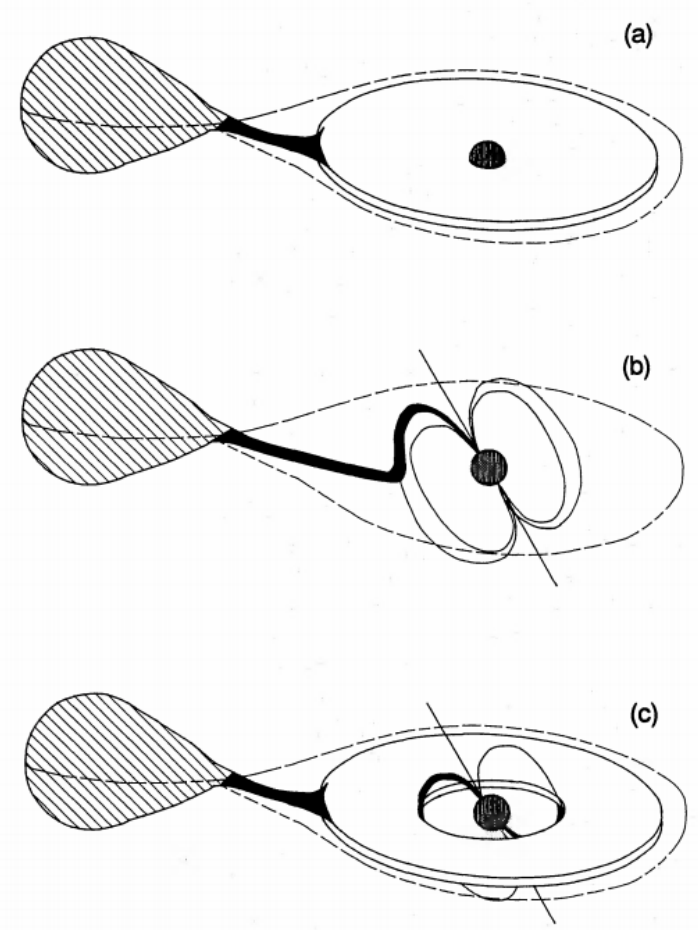

Fig. 2 Schematic of the different type of CVs. From top to bottom; a non-magnetic CV with an accretion disc (a), a polar (AM Her type systems) in which the accretion disc is prevented from forming (b) and an intermediate polar (DQ Her type systems) in which the accretion disc is disrupted (c) [6].

arc-like accretion regions near the WD poles, similar to the auroral ovals on the Earth and Jupiter.

However, there are systems that do not fit into the standard picture of mCVs. AE Aqr was originally classified as an IP [8] but there are a number of unusual observational features that are not naturally explained by this model It is known to switch between the flaring and quiescent states irregularly, which distinguishes it from other mCVs. Studies of the $33 \mathrm{~s}$ WD pulsations in the optical/UV [9] and X-rays [10, 11] show no correlation between their amplitudes and the flaring of the system, which means that the flares are not related to the process of depositing material onto the WD. [9]. The Balmer emission lines are single peaked and produce Doppler tomograms that are not consistent with those of an accretion disc. [12]. Different models were proposed to explain the observational properties of AE Aqr. The first, a "magnetic propeller" model [13] suggests that the rotation rate of the WD decelerates by means of interaction between its fast rotating magnetosphere and the material inflowing from the secondary. The second "pulsar-like white dwarf" model [14] show that the observed 
braking of the white WD can be explained in terms of the canonical pulsar-like spindown mechanism [14, 15]. Recently, a numerical propeller model of AE Aqr using axisymmetric magneto-hydrodynamic (MHD) simulations has been developed [16]. They suggest that an accretion disc forms around the WD that interacts with the magnetosphere of the star in the propeller regime. The model explains the rapid spin-down of the WD through the outflow of angular momentum from the surface of the WD into the wind and predicts that $\sim 85 \%$ of the inner disk matter is ejected into the conically-shaped winds. The model can also explain the low accretion rate onto the star if the radiative efficiency of accretion is $\sim 7 \%$,

\subsection{Models}

The optical flux and polarization of polars is highly modulated with orbital phase. These observations are explained by the cooling of the post-shock region through cyclotron emission which depends strongly on the viewing angle and the physical properties of the emitting region. Stokes techniques are consequently a powerful tool to diagnose the physical properties of the accreting region. An increase in circular polarization is observed when one looks down on the shock (parallel to the magnetic field lines), whereas an increase in linear polarised flux is observed when one views the side of the shock (perpendicular to the magnetic field lines).

The first important step in modelling of polar observations were performed in the 1980's and assumed the emitting region to be a 1-D point source. [17, 18, 19]. Wickramasinghe \& Meggitt (1985) present a grid of fluxes, and linear and circular polarization as a function of viewing angle and harmonic, which can be compared to optical data of polars. However, there is observational evidence of extended emitting regions in polars and so the models were improved to 2-D by using a sum of distinct point sources to represent the emitting region [20, 21].

More sophisticated models and fitting techniques were then developed referred to "Stokes imaging" [21] was the first objective and analytical technique that robustly models the cyclotron emission and maps the accretion zones in magnetic mCVs. This technique allowed objective mapping of the cyclotron-emission regions in terms of location, size and density structure. Stokes imaging uses a cyclotronemission model and a grid of cyclotron-emission calculations which include cyclotron and free-free opacities [19]. The emission from a single emission spot on the surface of the white dwarf is modelled by first calculating the local magnetic field strength and subsequently the local optical depth parameter. The cyclotron emission grids are then used to produce variations in the polarized emission as a function of phase. The emission from extended sources is modelled by summing the components of many such emission spots. Projection effects of extended regions are also taken into consideration. Fig. 3 shows the model fit from Stokes imaging for the IP V347 Pav, where Stokes imaging reproduced the main polarization morphology [22]. The main emission region was found to consist with two relatively higher density regions centred on the magnetic equator. The system inclination derived is 68 
degrees and the magnetic field strengths are 15 and 20 MG for the upper and lower poles respectively. Stokes imaging has been advanced by the addition of more realistic stratified accretion shock model in order to calculate cyclotron spectra, and the use of Stokes imaging in combination with Doppler and [23] Roche tomographic [24] techniques, to gain insights into magnetic accretion [25].

A more detailed code has also been developed, which adopts a 3-D representation of the accretion column and WD, incorporates the radiative transfer of the cyclotron process in the post-shock region [26]. The model has been used to fit the optical near-IR circular polarimetric light curves of V834 Cen (see Fig. 4) and the best fit is consistent with previous parameter estimates and is able to reproduce the observed features.
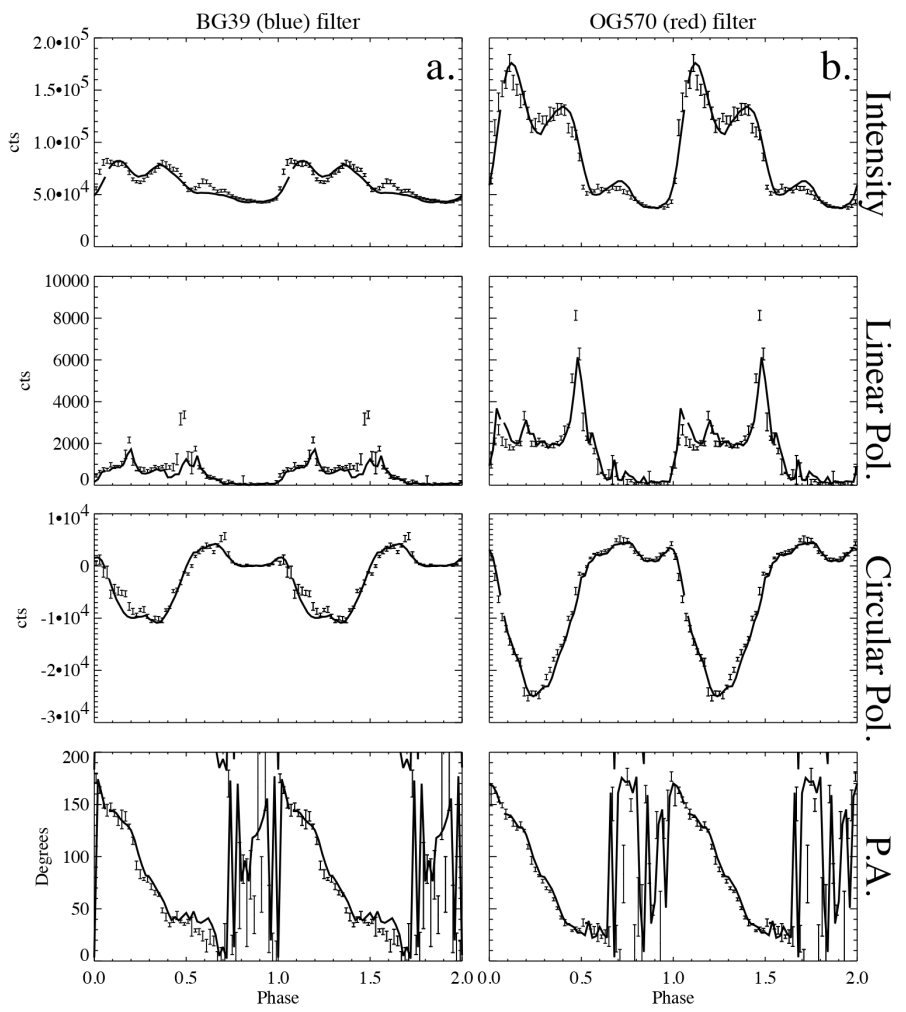

Fig. 3 The Stokes imaging optimized solution to the polarimetric observations V347 Pav. Solid curves are the model fit to the blue (a) the red (b) data. The imaging predicts that the bright-phase emission arisws from a large region towards the lower hemisphere, and the faint-phase emission from a single smaller region in the upper hemisphere of the WD [22]. 

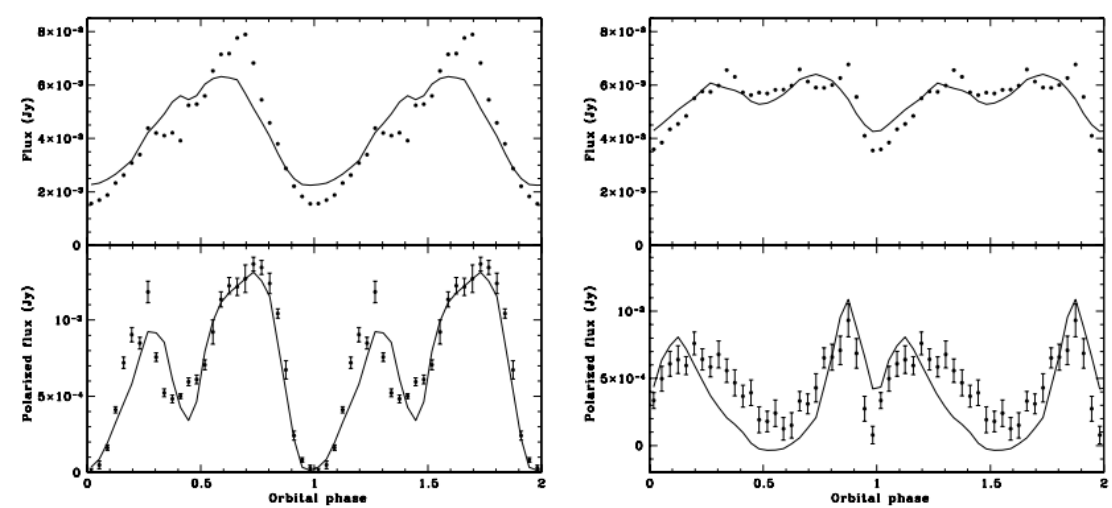

Fig. 4 Best fit to the $V$-band (left) and $J$-band (right) circular polarization light curves V834 Cen of using the CYCLOPS [26].

\subsection{Cyclotron spectroscopy}

Cyclotron spectroscopy is a useful method for extracting on strength of the magnetic fields in polar and IPs. The characteristic feature of polars is the presence of strong linear and circular polarization at optical and near-IR wavelengths. The polarized emission is due to cyclotron origin and arises from accretion shocks near the surface of the high magnetic field WD. The fundamental cyclotron frequency usually lies in the IR (from $\sim 2-10 \mu \mathrm{m}$ ). Indeed, the cyclotron radiation observed in the optical/near-IR consists of the harmonics of the fundamental cyclotron frequency. The spacing between successive cyclotron humps allows one to estimate the magnetic field strength of the cyclotron emission, because cyclotron emission intensity and polarization depend on the harmonic $n\left(=\omega / \omega_{c}\right)$ of the fundamental cyclotron frequency $\omega_{c}$ [19, 27]. The position of the $n_{t h}$ harmonic $\lambda_{n}$ of the cyclotron emission from a region with magnetic field $B$ and low electron temperatures $\left(T_{e}<10 \mathrm{keV}\right)$, as a first approximation is given by

$$
\lambda_{n} \sim \frac{10,710}{n}\left(\frac{10^{8}}{B}\right) \AA
$$

Cyclotron line emission has been observed in a number of polars, where cyclotron harmonics ( $n \sim 3$ to 8$)$ are seen in the optical/near-IR spectra. W Pup, [28], UZ For [29], MR Ser [30], DP Leo [31] ST LMi [27] and EF Eri [32].

The observed circular polarized flux is independent of other sources of nonpolarized light (e.g. accretion stream, disc or WD photosphere) and therefore is very useful for comparing with existing cyclotron models. The broadband circular polarized flux spectrum gives estimates to the magnetic field in the cyclotron emission region, as cyclotron emission intensity and polarization are characteristic to each harmonic [19]. An example of the comparison of the observed circular polarized 


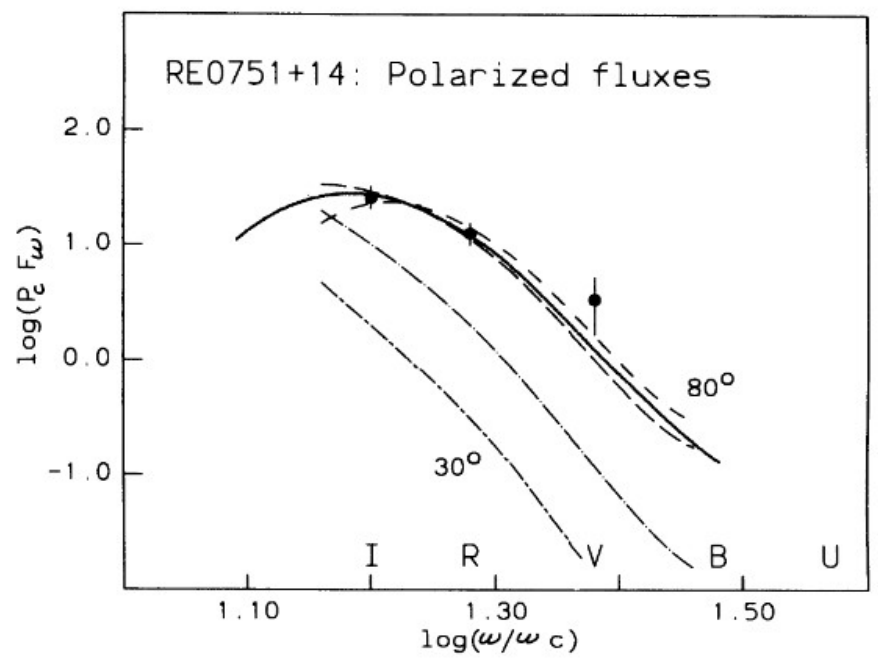

Fig. 5 Observed peak circular polarized flux compared with cyclotron models with different magnetic field strengths [33]. (C)AAS. Reproduced with permission.

flux spectrum with cyclotron models is shown in Fig. 5. IPs are known to be circularly polarised at a level $\sim 1-3 \%$ [2]. Magnetic field measurements for IPs which do not show measurable photospheric Zeeman features are difficult, but from the polarized flux spectrum, estimates have been made for PG Gem (8-18 MG [33]); PQ Gem (9-21 MG [34, 35]), V2400 Oph (9-20 MG [36]), RX J2133.7+5107 (>20 MG [37]) and V405 Aur ( 30 MG [38]). These values are similar to the magnetic field strengths measured in polars, and suggest that some of the polarized IPs may evolve into polars [39].

\subsection{Time-resolved polarimetry of SW Sex systems}

SW Sex stars are a sub-class of CVs that possess common but inexplicable features, very different from other CVs [40]. Their light curves show deep eclipses suggesting a high-inclination accretion disc but their emission lines show single-peaked profiles that do not share the orbital motion of the WD and they exhibit transient absorption features as well as shallow eclipses. This is contrary to the deeply-eclipsing, doublepeaked profiles one would expect for high-inclination systems [23]. Many models have been proposed to explain the behaviour of the SW Sex systems. Models include gas-stream overflow, magnetic propeller accretion, and self obscuration by the disc rim (for details see [41]). Dhillon et al. (2013) have proposed that a combination of dominant bright-spot emission and a self-occulting disc can explain the observed properties. 


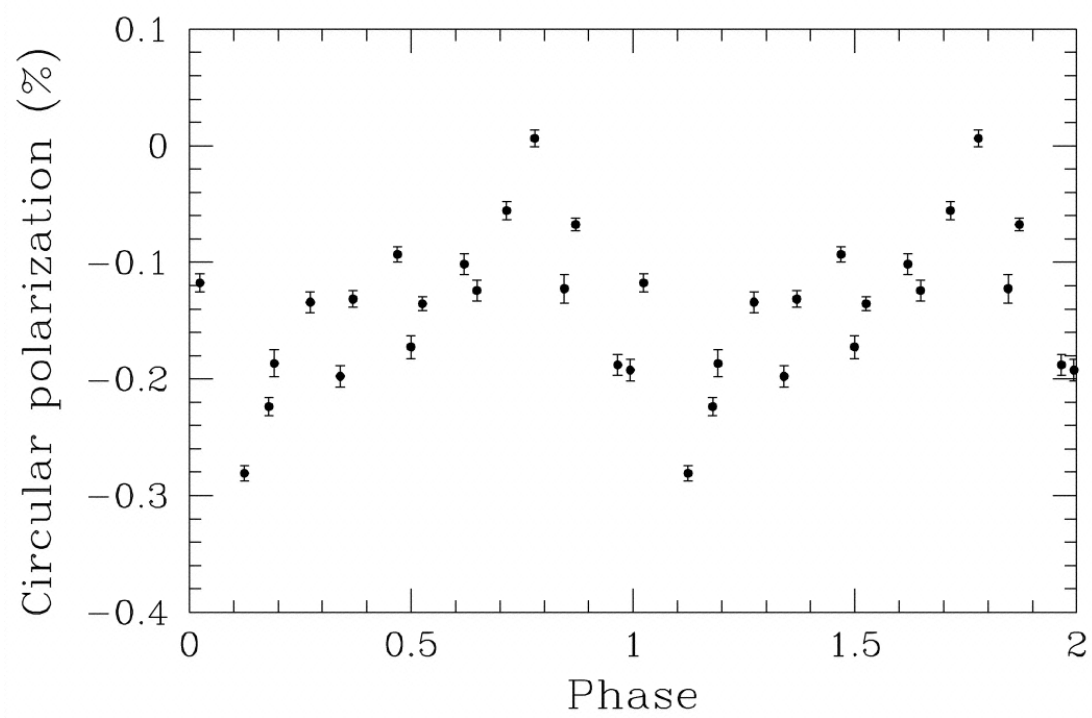

Fig. 6 Modulated circular optical polarization in LS Peg. The period of the modulation is in phase with the spin period of the WD [42]. (C)AAS. Reproduced with permission.

A magnetic origin for the SW Sex systems has been claimed by several authors (e.g. see [43]). Although the observation of modulated circular polarisation is an unambiguous proof as it implies the direct detection of a relatively strong magnetic field, the presence of coherent pulsations related to the WD spin period and/or the orbital period also provides strong evidence for the presence of a magnetic WD. Modulated circular polarization has been observed in the non-eclipsing systems LS Peg (see Fig. 6, V795 Her and RXJ1643.7+3402 [42, 44, 45]. In LS Peg Xray pulsations associated with the spin period of the WD is observed at the same polarimetric frequency [46], whereas in RXJ1643.7+3402 the circular polarization period is interpreted as half the beat period between the WD spin period and the orbital period [45]. To some extent the observed circular polarization frequencies can be explained in terms of a magnetic model in which the overflowing gas stream crosses the WD's magnetosphere at approximately the corotation radius [42, 45].

\subsection{A WD pulsar}

The discovery of pulsar-like behaviour in the close binary system AR Scorpii (hereafter AR Sco) demonstrates that WDs can exhibit many of the same characteristics as neutron star (NS) pulsars. Highly pulsed non-thermal emission is observed from radio to the UV arising from the spinning WD [47]. 


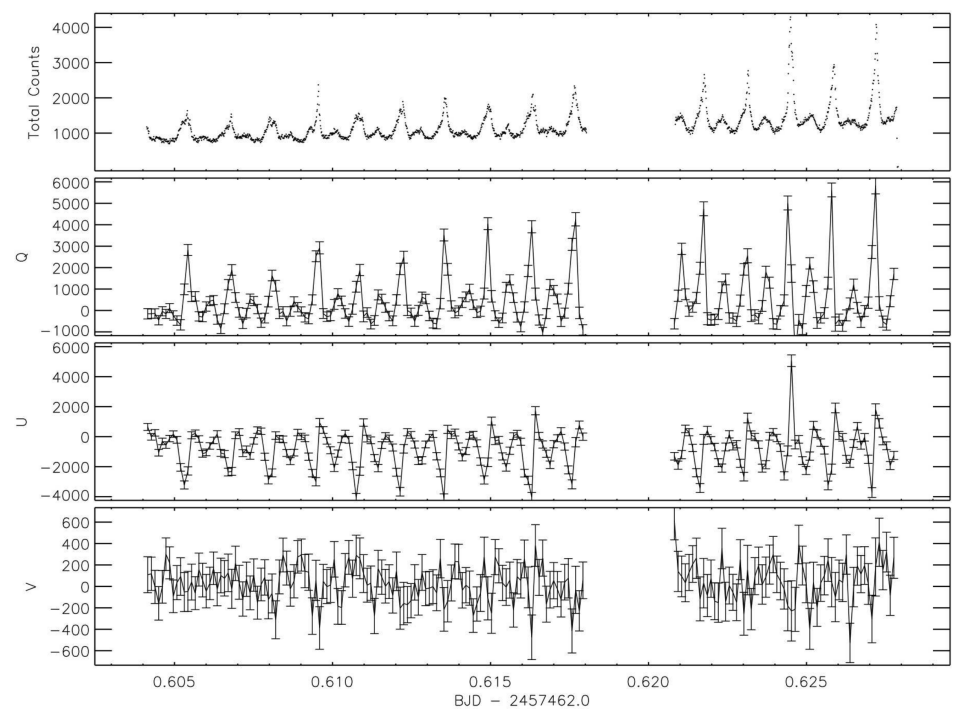

Fig. 7 Broad band photopolarimetry of AR Sco. From the top to bottom, the total intensity and the Stokes Q, U \& V values [49].

These pulsations are seen predominantly at the $118 \mathrm{~s}$ beat period between the 117 $\mathrm{s}$ spin period and the $3.6 \mathrm{~h}$ orbital period of the binary. The strong spin modulated emission arising from the WD plus the dominant non-thermal nature of the spectral energy distribution suggests that magnetic interactions power the emission in AR Sco [47, 48], similar to what is observed in pulsars. Further evidence of pulsar behaviour is given by polarimetric observations [49]. Strong linear polarization (up to $40 \%$ ) that varies on both the spin period of the WD and the beat period between the spin and orbital period has been detected as well as low-level circular polarization $(\sim$ few $\%)$. The characteristics of the polarized and non-polarized emission are explained in terms of synchrotron emission from two different regions, one associated with the rotating WD magnetic field and the other as a result of MHD interactions with the M-dwarf companion star. Unlike typical CVs there appears to be little accretion on to the WD from its M-dwarf companion star [47], which means that gravitational potenitial energy is not the source of the emission. AR Sco is the first example of a WD pulsar. because the pulsed luminosity of AR Sco is powered by the spin-down of the rapidly rotating highly magnetized $(<500 \mathrm{MG})$ WD [49].

\section{X-ray binaries}

$\mathrm{X}$-ray binaries (XRBs) are binary systems in which a compact object, a black hole $(\mathrm{BH})$ or a NS, accretes matter from a companion star. During the outburst of a black 


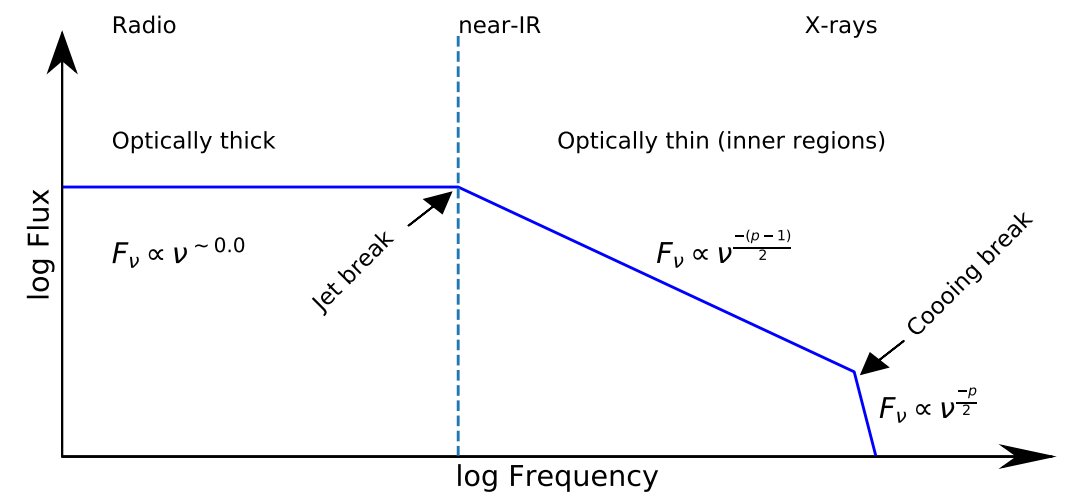

Fig. 8 A sketch of spectrum of a steady, hard-state jet. The radio to near-IR spectrum is due to a $\sim$ flat, self-absorbed optically thick synchrotron spectrum. The key spectral point is the break from optically to optically thin synchrotron emission, between the mid- to near-IR wavelengths.

BH-XRBs, continuously replenished synchrotron-emitting "compact" jets are commonly produced during the hard spectral state (hard-state; [50]). (For descriptions of X-ray states see [51]). The hard-state usually occurs at the beginning and end of an outburst, with X-ray luminosities $>10^{33} \mathrm{erg} \mathrm{s}^{-1}$ and up to $\sim 1-5 \%$ of the Eddington luminosity (e.g. [52, 53]), with a highly variable hard power-law X-ray spectrum that originates from the inner, radiatively inefficient accretion flow close to the black BH [54]. A classical "flat spectrum" radio jets (similar to those seen in AGN) are commonly observed during the hard-state, when the accretion flow structure allows a large-scale height magnetic field. In the softer X-ray states jets are dramatically quenched at radio frequencies [55, 56, 57] because the geometrically thin disc suppresses any large-scale vertical field [58].

Few studies have attempted to uncover the polarimetric signature of the optically thin synchrotron emission from compact jets in XRBs. The polarization originates close to the base of the jet where the magnetic field should have higher level of ordering over the smaller emission region compared to further out in the jet [59]. Polarimetric measurements of the optically thin power law synchrotron emission provides a powerful tool to uncover the nature of the magnetic field structure in this region, because it is associated with the start of the particle acceleration in the jet [60], which is important for models and simulations of jet production.

\subsection{The spectral energy distribution}

The spectral energy distribution of an XRB can be described by thermal emission from a companion star and accretion disc, synchrotron emission from a jet and a 


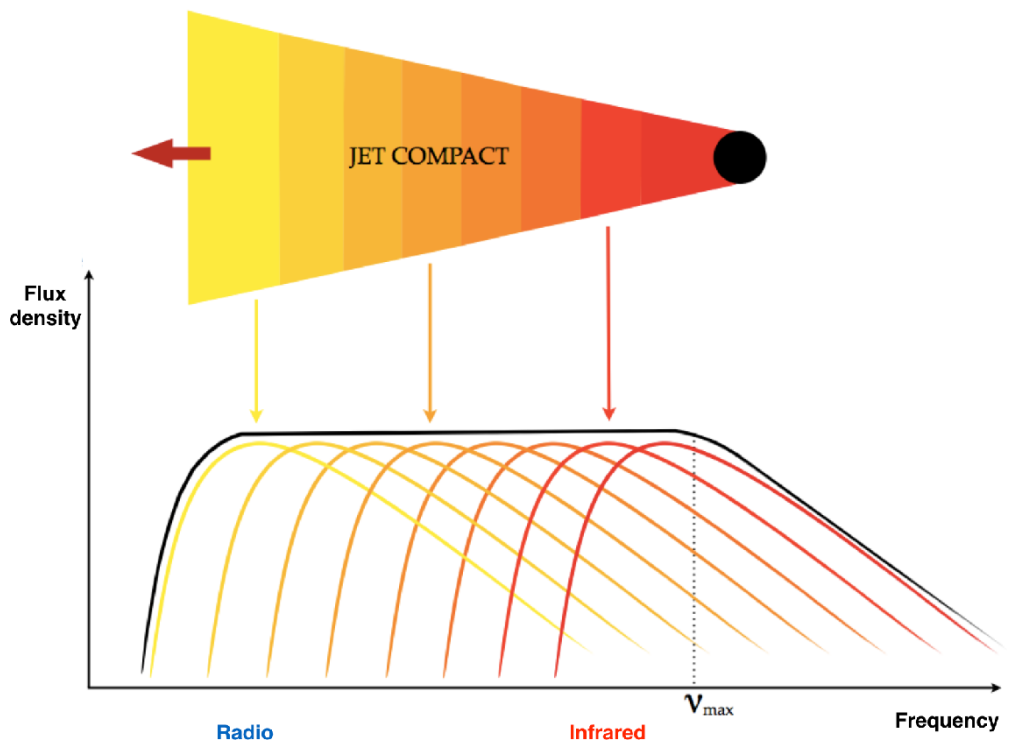

Fig. 9 The spectral energy distribution of the jet which is made up of different synchrotron emitting regions along the length of the jet [61].

Comptonized corona from the disc. At optical and IR wavelengths the companion star dominates the optical/near-IR emission whereas the accretion disc dominates at UV to X-ray wavelengths. The radio to IR spectrum of an XRB in the hardstate is due to a $\sim$ flat, self-absorbed optically thick synchrotron spectrum with a spectral index of $\alpha \sim 0.0$ to +0.5 , where $F_{v} \propto v^{\alpha}$ [55, 62, 63] (see Figs. 8,9 and 10 , which is the principal signature of the presence of a conical, collimated jet in black BH-XRBs. It typically extends at down to at least to millimeter wavelengths and is explained as being due to the superposition of synchrotron-emitting lepton particle distributions at different radii from the compact object [59, 64, 65]. (see Fig. 9). The higher energy synchrotron emission arises from a small, dense region of the jet, close to the location where the jets are launched, near the compact object [66]. Since $\alpha>\sim 0$, the bulk of the radiative power of the jet resides in this higher energy emission and so at some frequency ( $\sim$ IR wavelengths) there is a break in the jet spectrum (e.g. [67, 68]). Above this break frequency, the flat spectral component breaks to an optically thin spectrum corresponding to the point at which the entire jet becomes transparent, close to the jet base in the post-acceleration plasma (see Fig. 99. In addition, there is a cut-off in the jet spectrum at higher energies, in the X-ray region [63, 69] (see Fig. 8).

The transition between the optically thick and thin part of the spectrum occurs at the jet break frequency, which usually lies in the mid-IR to near-IR, where the optically thin synchrotron emission is observed, resulting in a power law with index $-1<\alpha<-0.5$ [70, 71] (see Fig. 10]. The break frequency has been detected in a 


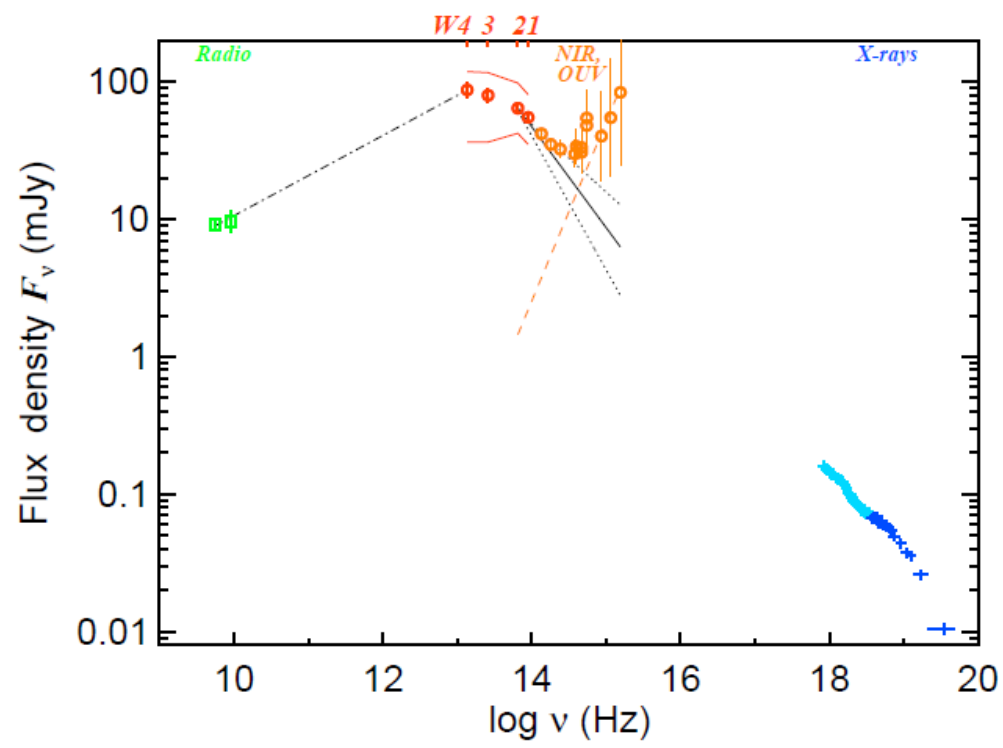

Fig. 10 The spectral energy distribution of GX 339-4. The spectrum breaks (jet break) from optically thick to optically thin in the mid-IR [70]. (C)AAS. Reproduced with permission.

the BH candidates GX 339-4 [67, 70, 72], XTE J1118+480 [73], XTE J1550-564 [74], V404 Cyg [75, 76], MAXI J1836-194 [71, 77], Cyg X-2 [78], in the quiescenct BH-XRBs, Swift J1357.2-933 and A0620-00 [79, 80, 81], and in the NS systems 4U 0614+091, 4U 1728-34, and Aql X-1 [82, 83, 84]. The steady jet spectrum evolves with the break frequency (from optically thick to optically thin) moving as a function of time and spectral state as the electron population cools [77]. During an $\mathrm{XRB}$ outburst, as the X-ray spectrum of the source softens, the jet break frequency moves from the near-IR to radio frequencies and then return to the near-IR at the end of the outburst, when the spectrum gets harder [71, 84]. In BH-XRBs, as the source softens during state transitions, the jet break is observed to shift from the IR to millimetre frequencies [85]. In the soft X-ray state, no core jet is detected. The structure of the accretion flow is governed by the presence of large-scale height magnetic fields that determine the position of the break frequency. Since the size of the jet-emitting region scales inversely with the frequency [59] the break frequency gives information on the size of the base of the jet where the particles are accelerated [74, 86].

Fast sub-second optical/near-IR variability and correlations have also been observed in various systems [87, 88, 89, 90]. The most likely explanations is a jet origin, because the variability has a spectrum consistent with optically thin synchrotron emission [91, 73], which is stronger in the near-IR [92, 90], maybe due to variable synchrotron emission of the X-ray corona [93]. 

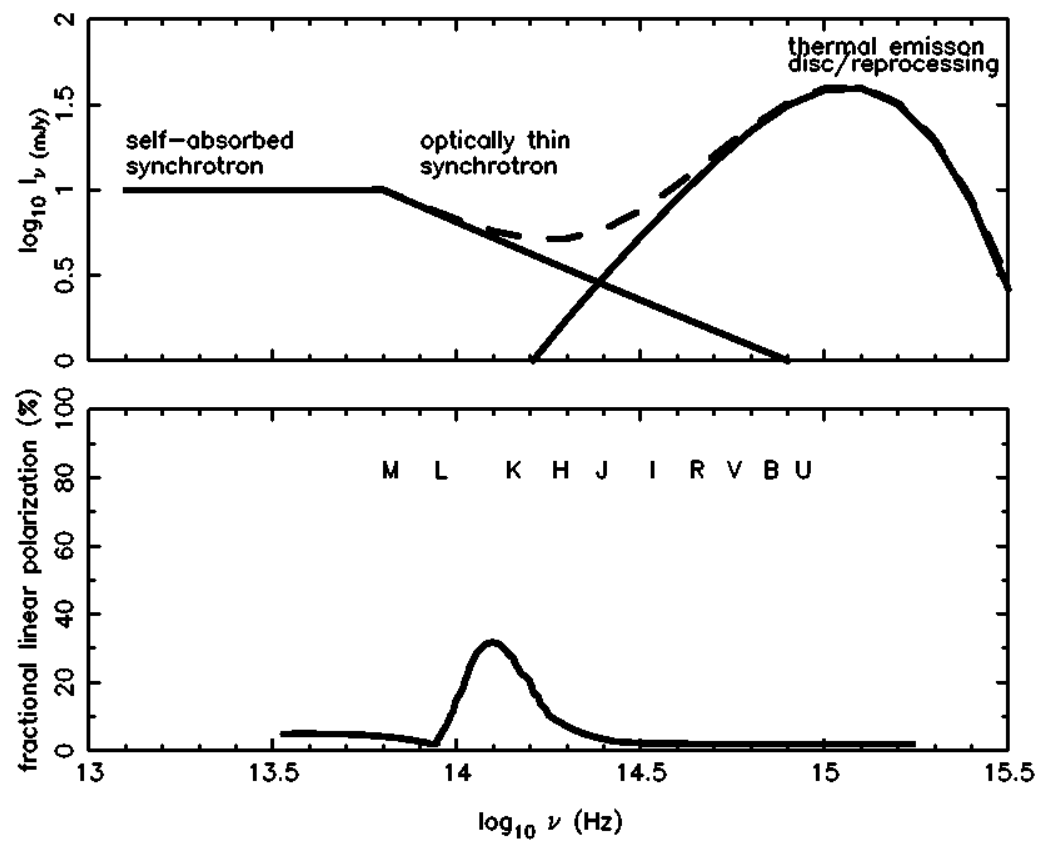

Fig. 11 The expected linear polarization signature in the optical and near-IR spectrum of XRBs. The key spectral points are the break from optically thick (low polarization $<10 \%$ ) to optically thin (high polarization $<70 \%$ ) synchrotron emission, and the point at which the thermal (lowpolarization) emission begins to dominate over the jet. These effects should combine to produce a narrow spectral region with relatively high linear polarization arising from close to the base of the jet [94].

\subsection{The polarization spectrum of XRBs}

Shahbaz et al. (2008) outlined the expected intrinsic linear polarization signature of $\mathrm{XRBs}$ in the optical/near-IR region. The optical and IR light from XRBs is polarized due to intrinsic polarization of the emitting photons, or by the (Thompson or possibly Rayleigh) scattering or absorption of unpolarized photons. Hydrogen in the disc is totally ionised and a low linear polarisation is expected in the optical due to Thomson scattering of emitted unpolarised radiation with free electrons in the disc. In the case of the persistent XRBs where the accretion disc dominates the optical light, the linear polarization has a component that is produced by electron scattering by plasma above the accretion disc. Although a strong interstellar component is present, for Rayleigh and Thompson scattering processes, the level of linear polarization decreases as a function of increasing wavelength. Electron scattering of the disc radiation by the secondary star is insignificant, because the secondary stars in XRBs have low surface temperatures and therefore low free electron densities. Polarization of the optical light due to absorption by interstellar dust is inferred by 
the characteristic constant wavelength-dependent polarization, given by Serkowski's law [95, 96]. There also exists the possibility of a large fractional linear polarization (FLP) from optically thin synchrotron emission. Indeed observations and theoretical results suggest a break between optically thick and thin emission occurs around the near-IR band (e.g., see [67, 75] and Fig. [10).

Optically thick synchrotron emission has a maximum linear polarization of $\sim 10 \%$, whereas optically thin synchrotron emission can have a FLP as high as $70 \%$ [97]. Two polarization signatures are expected from the jet. For the optically thick part of the spectrum, which results in the flat spectral component observed in the hard X-ray state [55], no more than a few \% polarization is expected. At long wavelengths there should be $\sim 1 \%$ polarization from the self-absorbed jet and at short wavelengths a comparable level will be measured due to scattering in the accretion flow. However, in the relatively narrow spectral region in which optically thin synchrotron emission dominates, one expects a strong signature that initially rises to longer wavelengths as the relative jet/disc fraction increases (see Fig. 11].

\subsection{The theoretical polarization spectrum of the jet}

Relativistic electrons moving in a large-scale and ordered, uniform magnetic field produce synchrotron radiation. If the relativistic electrons produced by the firstorder Fermi acceleration have a non-thermal energy distribution, the synchrotron radiation is linearly polarized. The linear polarization spectrum of optically thin synchrotron emission from an relativistic electrons in a uniform magnetic field was first treated by Westfold (1959). Subsequent works have extended these ideas to include power-law electron energy distributions and non-uniform magnetic fields (e.g. [99, 100, 101]). Indeed, Bjornsson et al. (1982) derived the more general case for optically thin synchrotron emission from an electron population and magnetic field with an arbitrary distribution of energies and configuration, respectively. They showed that the expected FLP is given by

$$
\mathrm{FLP}_{\text {thin }}=\mathrm{f} \frac{\mathrm{p}+1}{\mathrm{p}+7 / 3}=\mathrm{f} \frac{1-\alpha_{\text {thin }}}{5 / 3-\alpha_{\text {thin }}}
$$

where $\alpha_{\text {thin }}[=(\mathrm{p}-1) / 2]$ is the spectral index of the optically thin synchrotron emission of the form $\mathrm{F}_{v} \propto v^{\alpha}, f$ represents the ordering of the magnetic field and $p$ is the electron energy distribution $\left(\mathrm{N}(\mathrm{E}) \mathrm{dE} \propto \mathrm{E}^{-\mathrm{p}} \mathrm{dE}\right)$ [102]. The parameter $f$ takes values between 0 and 1 , where 0 represents non-uniform, no net field orientation and 1 a perfectly uniform, fully aligned field. If the field is tangled, the differing angles of the polarized light suppress the observed, average polarization. In the case of a fully ordered field [102, 103] the maximum polarization strength is $\sim 70-80 \%$ and is dependent only on the degree of ordering of the field and the energy distribution of the electron population. However, if the spectral index is steeper the polarization can be higher. The electric (polarization) vector is perpendicular to the projection of the magnetic field on the plane of the sky. 

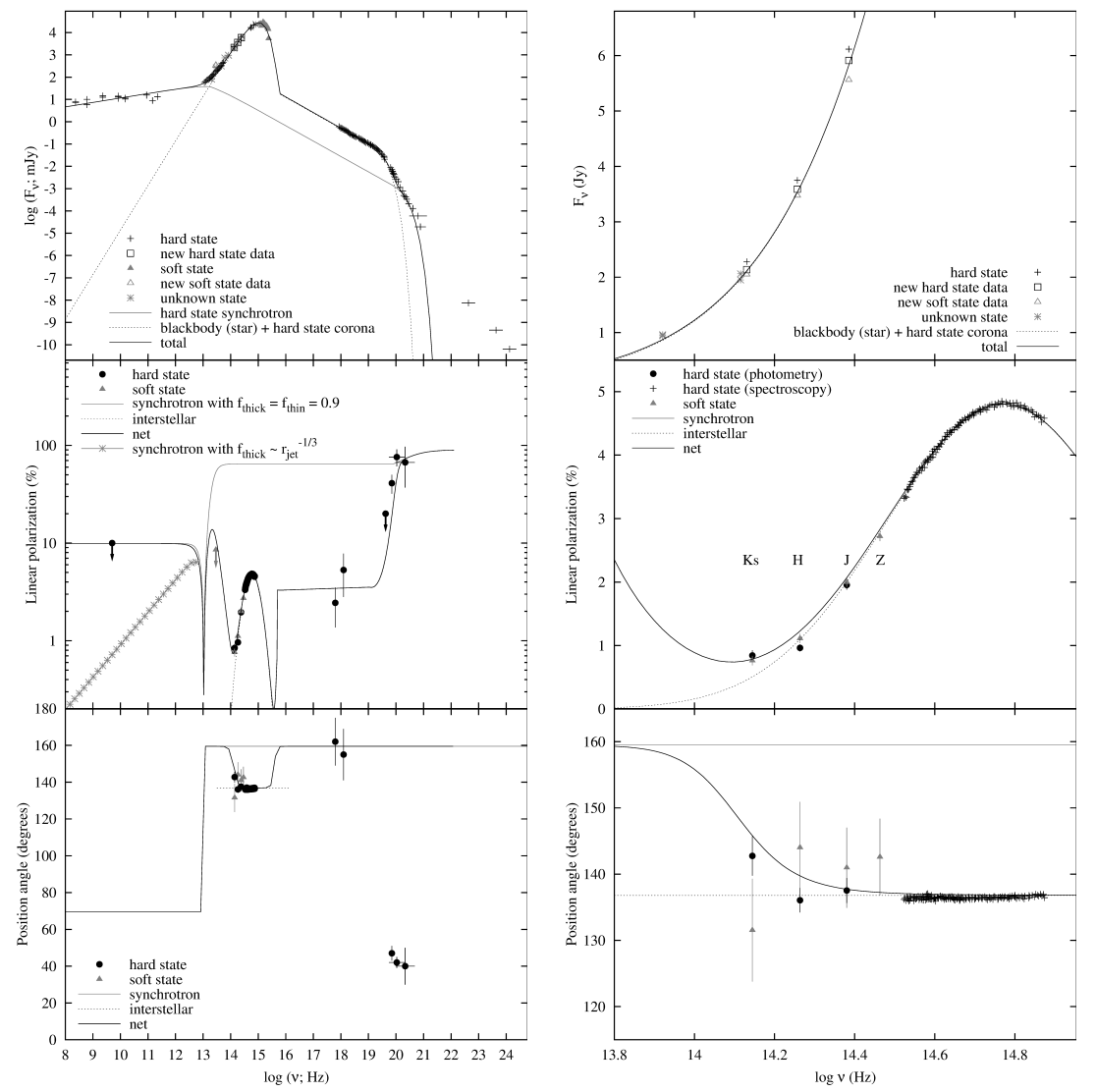

Fig. 12 Left: Radio to $\gamma$-ray flux density (top), linear polarization spectrum (middle) and polarization position angle (bottom) spectrum of $\mathrm{Cyg} \mathrm{X-1} \mathrm{Right:} \mathrm{the} \mathrm{same} \mathrm{as} \mathrm{the} \mathrm{left-hand} \mathrm{panels} \mathrm{but} \mathrm{just}$ the near-IR/optical region [98].

For a synchrotron source with a power-law electron energy distribution the FLP is frequency-independent. However, a high energy cutoff in the electron distribution results in frequency-dependent FLP [104]. Above the high energy cutoff frequency the polarization is given by

$$
\mathrm{FLP}_{\mathrm{cut}}=\mathrm{f} \frac{1-\alpha_{\mathrm{cut}}}{5 / 3-\alpha_{\mathrm{cut}}}
$$

where $\alpha_{\text {cut }}$ is the spectral index defined by power-law emission model. The above situations corresponds to a highly idealized situation, assuming optically thin plasma, isotropic distributions of electrons, perfectly ordered homogeneous magnetic fields, and the absence of substantial perturbations. A major modification occurs for optically thick plasma where each light ray experiences multiple scattering events. 
For optically thick, absorbed synchrotron emission $\left(F_{v} \propto v^{5 / 2}\right)$ the net polarization spectrum is given by

$$
\mathrm{FLP}_{\text {thick }}=\mathrm{f} \frac{3}{6 \mathrm{p}+13}
$$

with a polarization position angle (PA) that differs by 90 degrees to that of optically thin synchrotron polarization [105, 106, 97, 101]. The polarization is less polarized than optically thin synchrotron [97]. For optically thin synchrotron emission with a typical spectral index of $\alpha_{\text {thin }}=-0.7(p=2.4)$, FLP $=11 \%$. The electric vector in this case is parallel to the projected magnetic field. If the level of ordering of the magnetic field is constant along the jet, with a constant PA, then Equation 4 can be used to describe the polarization expected from a flat/inverted optically thick jet spectrum.

An outlined in detail in Russell \& Shahbaz (2014), equations 2 to 4 can used to predict the FLP as well as the polarization PA of the synchrotron emitting jet spectrum. Given that different components produce emission with different FLPs and PAs, it is necessary to calculate the Stokes parameters $Q$ and $U$ for each component at each frequency from the known FLP and PA values, using the standard equations relating polarization and PA.

\subsection{Polarization in X-ray persistent XRBS}

At optical/near-IR wavelengths, components such as the accretion disc and companion star often dominate the spectrum of BH- and NS-XRBs, suppressing any synchrotron contribution to the polarization. However, when the synchrotron makes a strong contribution, intrinsic polarization is detected [107, 94, 108, 109, 74, 110]. Intrinsic near-IR polarization has been detected in three $\mathrm{BH}-\mathrm{XRBs}$ during outburst, GRO J1655-40, XTE J1550-564 and GX 339-4, all during the hard-state when jets are expected to be launched [108, 74, 109].

In GX 339-4, when the polarization is strong the polarization angle is perpendicular to the known jet axis, implying a magnetic field that is parallel to the jet axis (see Fig. 14). The low levels of polarization measured ( 1-7 \%) and variability imply a tangled, rapidly changing magnetic field geometry near the base of the jet [108, 109], Mid-IR variability of GX 339-4, also show that either the acceleration region near the base of the jet, or its magnetic field strength, are changing on timescales of minutes [70], the same timescale as the polarization variability. During the bright outburst of V404 Cyg in 2015, flares of optical polarization were also detected and interpreted as synchrotron emission from a variable jet [112, 111]. Time-resolved optical polarimetry revealed a polarization flare which decayed fairly smoothly from 4.5 to $3.5 \% 20 \mathrm{~min}$, with a stable PA that is aligned with the resolved radio jet. This implies that the electric field vector near the base of the jet in V404 Cyg is on average approximately parallel to the jet axis and that the magnetic field is orthogonal to the jet axis. This may be due to the compression of magnetic 


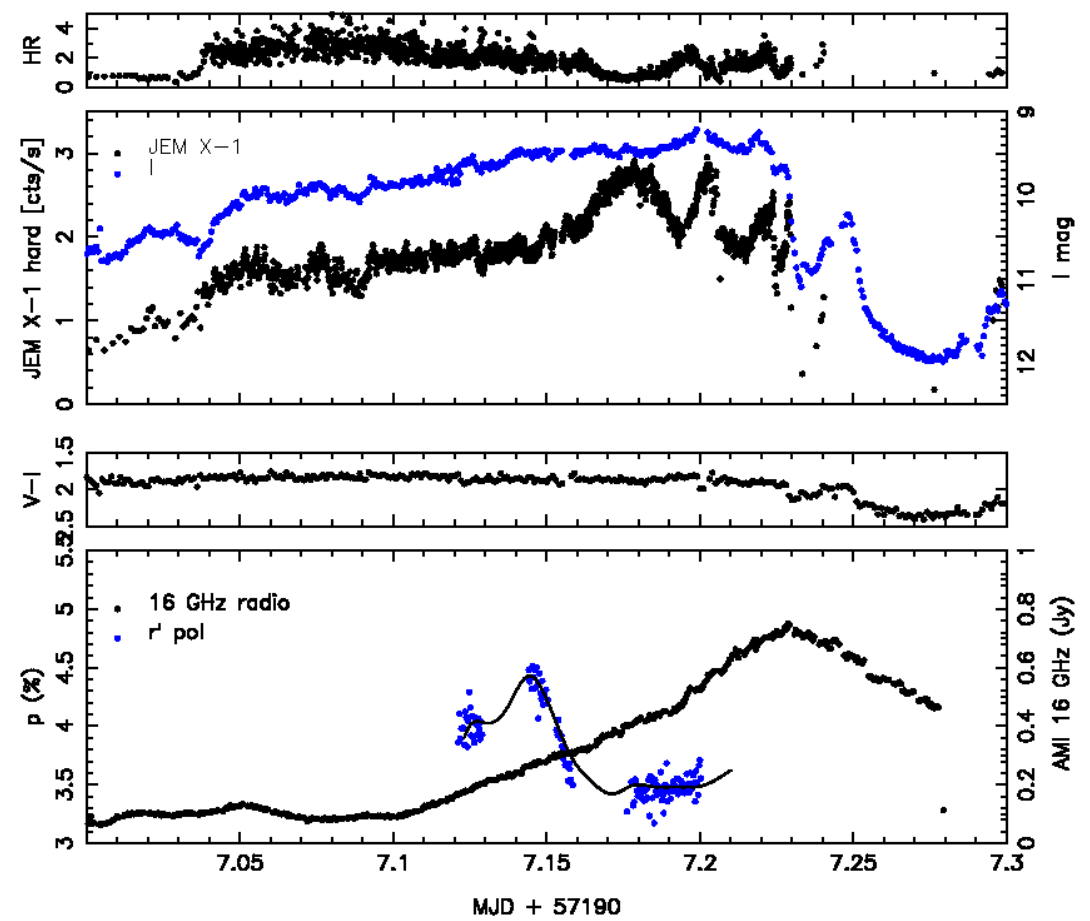

Fig. 13 The X-ray, optical and radio light curves of the V404 Cyg during the outburst in 2015. around MJD 57197. From top to bottom: INTEGRAL JEM-X X-ray hardness ratio; INTEGRAL JEM-X X-ray count rate (black dots) with the AAVSO I-band light curve (blue dots); AAVSO optical colour; and the AMI-LA $16 \mathrm{GHz}$ radio data with the $r^{\prime}$-band polarization light curve (blue dots) [111].

field lines in shocks in the flow, resulting in a partially ordered transverse field. The polarization flare also occurs during the initial rise of a major radio flare event that peaks later, and is consistent with a classically evolving synchrotron flare from an ejection event (see Fig. 13.

To date, the most detailed polarization spectrum has been obtained for Cyg X-1. Russell1 \& Shahbaz (2014) modelled the broadband, radio to $\gamma$-ray flux spectrum and polarization spectrum of $\mathrm{Cyg} X-1$ in the hard-state, with a simple phenomenological model consisting of a strongly polarized synchrotron jet (see Sect.2.3 and an unpolarized Comptonized corona and a moderately polarized interstellar dust component (see Fig. 12). They were able to show that the origin of the $\gamma$-ray, X-ray and some of the IR polarization is the optically thin synchrotron power law from the inner regions of the jet.

In NS-XRBs, intrinsic near-IR or optical polarization at similar levels as the BHXRBs has detected in Sco X-1, Cyg X-2 and 4U 0614+09 [108, 94, 109, 110]. Shahbaz et al. (2008) performed near-IR spectropolarimetry of the XRBs Sco X-1 and Cyg X-2 and found them be intrinsically polarized, with an increasing FLP at lower 
frequencies, which is explained by optically thin synchrotron emission from a jet. The FLP observed is $\sim 1-10 \%$, with evidence for rapid variations in some sources on timescales of seconds-minutes [109]. This dependence on wavelength cannot be explained by scattering or interstellar dust absorption and it cannot explain the short timescale variability of the polarization observed Sco X-1 [109]. The PA is usually approximately orthogonal to the axis of the resolved radio jet, which implies the magnetic field is parallel to the jet axis. In general, the observations of most XRBs are consistent with a variable, predominantly tangled magnetic field geometry, with field lines preferentially orientated along the jet axis (see Fig. 14). However, there are a few exceptions. Cyg X-1, has a highly ordered, stable magnetic field near the base of the jet that is perpendicular to the jet axis [98, 113]. V404 Cyg shows a low level polarization flare, with the magnetic field also perpendicular to the jet axis [111], which may be due to the compression of tangled magnetic field lines in shocks downstream in the flow or collisions with dense regions of the interstellar medium, resulting in a partially ordered transverse field.

\subsection{Polarization in X-ray quiescent XRBs}

$\mathrm{X}$-ray transients are a subclass of the XRBs which spend the majority of their time in a state of quiescence, accreting at very low X-ray luminosities $\sim 10^{30}-10^{34} \mathrm{erg} \mathrm{s}^{-1}$, explained by a truncated accretion disc with a radiatively inefficient accretion flow [54]. Although it is not really clear if quiescence is a separate state, or simply a lowluminosity version of the hard-state, but observations show that quiescent BH-XRBs
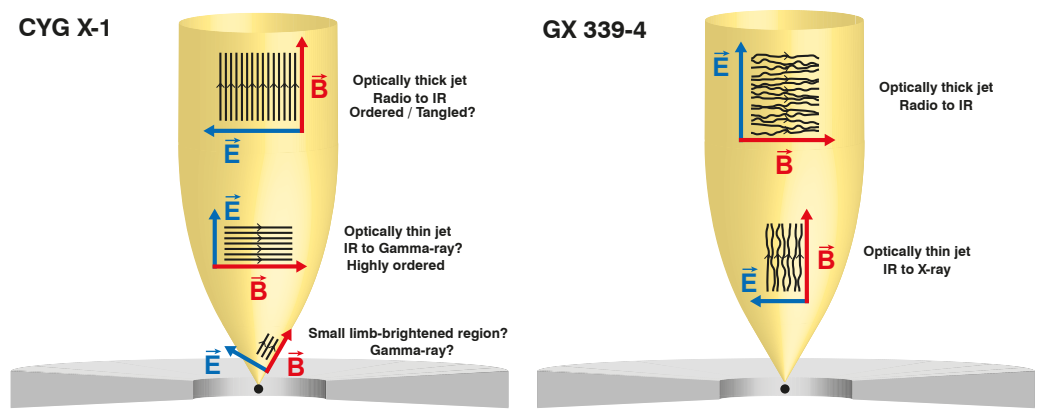

Fig. 14 A schematic diagram of the Cyg $X-1$ jet showing the orientation of the electric (from the near-IR observations) and magnetic field vectors (from observations of the radio jet) [98]. For synchrotron, the polarization PA traces the electric field vector in the emitting region, and the magnetic field vector differs by 90 degrees. In Cyg X-1 (left) a highly ordered magnetic field in the optically thin region near the jet base (as implied by the observations) and an ordered or tangled field in the large scale jet is shown. In other XRBs, such as GX 339-4, (right), the magnetic fields near the base of BH-XRB jets are moderately tangled, with a preferential orientation of the magnetic field along the jet axis. 
have softer X-ray spectra than hard-state systems (e.g. [114]) and outflows with weaker particle acceleration [115, 116]. There are a few radio detections of XRBs in quiescence, which include V404 Cyg [117], A0620-00 [118], XTEJ1118+480 [119], Swift J1753.5-0127 [120], 47 Tuc X9 [121], M62-VLA1 [122], MWC 656 [123] and M15 S2 [124], however, there is only one linear polarization constraint of the quiescent radio emission; $<2.11 \%$ in V404 Cyg [125]. A0620-00 and V404 Cyg are the only truly quiescent system with a robust radio detection that seem to follow the same radio/X-ray correlations seen in the hard-state [118].

Jets in quiescent XRBs are weaker than the brighter hard-state jets. The electron may be dominated by partially non-relativistic, perhaps Maxwellian energy distributions, leading to steeper spectral energy distributions than are typical for optically thin synchrotron emission [126, 127]. Evidence for synchrotron emission from the optical/near-IR spectral energy distribution in quiescence has been found in the BHXRBs A0620-00 [115, 128, 129, 80], Swift J1357.2-0933 [126, 79] and the NSXRB XTEJ1814-338 [130]. Near-IR polarimetric observations of two quiescent BH-XRBs has revealed the polarimetric signature of synchrotron emission [131]. In A0620-00, at the longest near-IR bands the PA of the excess $1.3 \%$ polarization changes significantly (see Fig. 15p. The PA of this weak synchrotron component, most likely from a jet, implies that the orientation of the magnetic field is approximately parallel to the radio jet axis [132]. In Swift J1357.2-0933 the near-IR flux variability imply a continuously launched, highly variable jet, which has a moderately tangled magnetic field close to the base of the jet. These results imply that weak jets in low luminosity accreting systems have magnetic fields which possess similarly orientations and tangled fields compared to the more luminous, XRBs at higher accretion rates (see Sect.2.4.

\subsection{Time-resolved polarimetry}

Phase-resolved linear polarimetry provides a unique probe of XRBs as one can obtain information on geometrical properties of the binary system from the light scattered by circumstellar material. In principle the orbital inclination (i) can be measured, which is essential in determining the components' masses, the fundamental parameters that determines a star's initial structure and subsequent evolution. Brown, McLean \& Emslie (1978) and Rudy \& Kemp (1978) have investigated the polarization produced by Thomson scattering in circumstellar envelopes in binary systems. They showed that the linear polarization has a characteristic variability around the binary orbit, associated with scattering in the stellar envelope that is distorted by the gravity of the compact object. One assume a circular binary orbit, scattering due to electrons and that the material is optically thin and co-rotates with the binary. The observed phase-resolved Stokes parameters $Q$ and $U$ light curves can be fitted with fit a truncated (first and second order) Fourier series, 


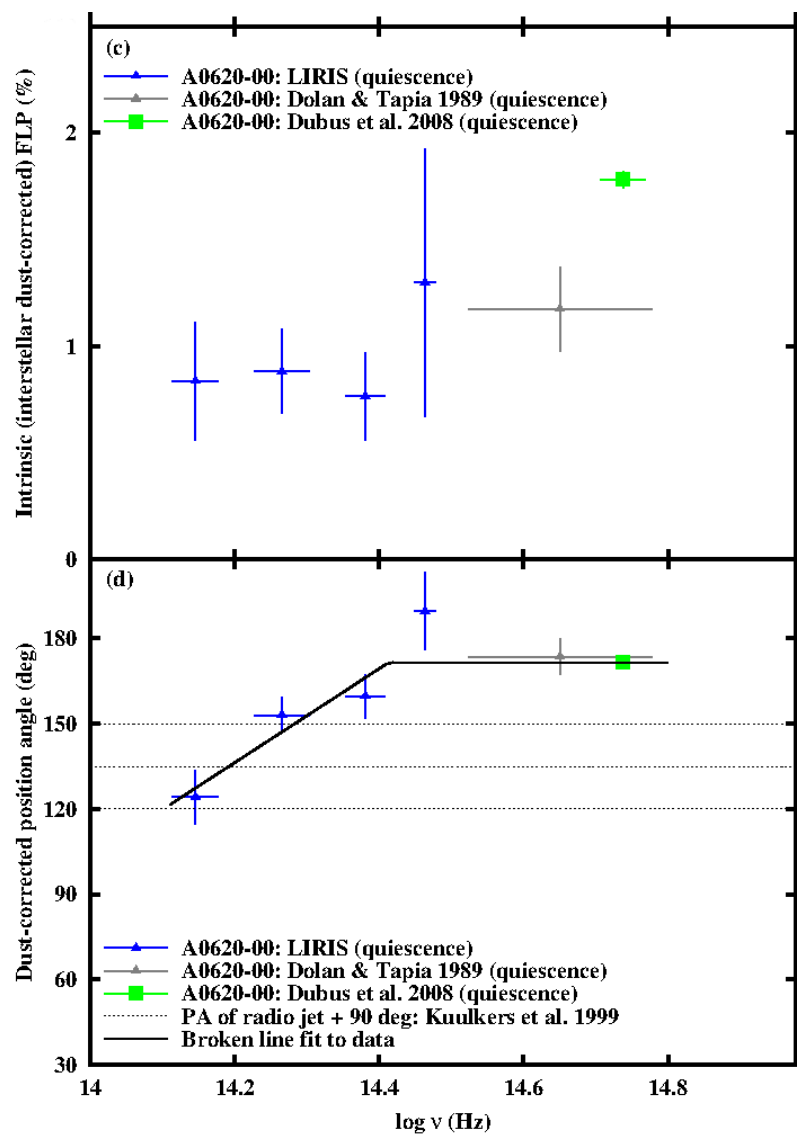

Fig. 15 The intrinsic (dust-corrected) near-IR linear polarization and position angle spectra of A0620-00 in quiescence [131]. The dust-corrected FLP of A0620-00 is $\sim 1-2 \%$ in the optical and $\sim 0.5-1.0 \%$ at near-IR wavelengths. The optical FLP is most likely due to Thomson scattering within the binary system [133], which is observed to vary with orbital phase [134]. Note the different PA in the $H$-and $K_{s}$-bands compared to in the optical.

$$
\begin{aligned}
& Q=q_{0}+q_{1} \cos \lambda+q_{2} \sin \lambda+q_{3} \cos 2 \lambda+q_{4} \sin 2 \lambda \\
& U=u_{0}+u_{1} \cos \lambda+u_{2} \sin \lambda+u_{3} \cos 2 \lambda+u_{4} \sin 2 \lambda
\end{aligned}
$$

where $\lambda=2 \pi \phi$ is and $\phi$ is the orbital phase. The variations are usually dominated by the second harmonics, which is commonly seen for binary systems showing polarization modulation. In the $Q-U$ plane the orbital phase-resolved polarization traces an ellipse twice per orbit. One can derive the orbital parameters either by studying the geometry of the $Q-U$ plane or by considering the Fourier coefficients. A least-square fits can be performed on the observed $Q$ and $U$ light curves to de- 
termine the coefficients and $q_{j}$ and $u_{j}(j=0-4)$, which can then be compared with theoretical expectations

$$
\begin{array}{r}
Q=\tau_{0}\left[\left(1-3 \gamma_{0}\right) \sin ^{2} i+\sin 2 i\left(\gamma_{1} \cos \lambda-\gamma_{2} \sin \lambda\right)\right. \\
\left.-\left(1+\cos ^{2} i\right)\left(\gamma_{3} \cos 2 \lambda-\gamma_{4} \sin 2 \lambda\right)\right] \\
U=2 \tau_{0}\left[\sin i\left(\gamma_{1} \sin \lambda+\gamma_{2} \cos \lambda\right)-\cos i\left(\gamma_{3} \sin 2 \lambda+\gamma_{4} \cos 2 \lambda\right)\right]
\end{array}
$$

where $\gamma_{i}$ are moments of the scatterers's distribution [133, 135]. The binary inclination is then given by

$$
\begin{aligned}
& {\left[\frac{1-\cos i}{1+\cos i}\right]^{2}=\frac{\left(u_{1}+q_{2}\right)^{2}+\left(u_{2}-q_{1}\right)^{2}}{\left(u_{2}+q_{1}\right)^{2}+\left(u_{1}-q_{2}\right)^{2}}} \\
& {\left[\frac{1-\cos i}{1+\cos i}\right]^{4}=\frac{\left(u_{3}+q_{4}\right)^{2}+\left(u_{4}-q_{3}\right)^{2}}{\left(u_{4}+q_{3}\right)^{2}+\left(u_{3}-q_{4}\right)^{2}}}
\end{aligned}
$$

Alternatively, in the $Q-U$ plane the orbital phase-resolved polarization traces an ellipse twice per orbit, and the eccentricity $e$ of this ellipse is related to the binary inclination by [136]

$$
\cos i=\sqrt{\frac{1-e}{1+e}}
$$

Hence, by determining the eccentricity of observed $Q-U$ ellipse, one can determine the binary inclination, independent of size, shape and position of the circumstellar region (see Fig. 16. An extension of the above model to an elliptical binary orbit can by found in [137] and [138]. Detailed accounts on polarimetric inclination determinations and their uncertainties are given by [139, 140, 141].

Time-resolved optical polarimetric studies of two XRBs has allowed one to estimate the inclination angle; $\mathrm{Cyg} X-1$ [142, 143, 144] and A0620-00 [134]. In Cyg X -1 a $\sim 5 \%$ optical linear polarization with an amplitude of $\sim 0.3 \%$ is observed, whereas in A0620-00 a 3\% linear polarization with a 2\% modulation is observed on the orbital period (see Fig. 16). Using the method outlined above, the inclination angle been estimated to be $i=62(+5,-37)$ degrees and $i=57(+20,-40)$ degrees for Cyg X-1 and A0620-00, respectively [143, 134]. The uncertainties in the values large mainly because of the poor phase-sampling and precision of the Stokes light curves.

Acknowledgements I would like to thank Dave Russell for the many discussions on the works presented in this review. 

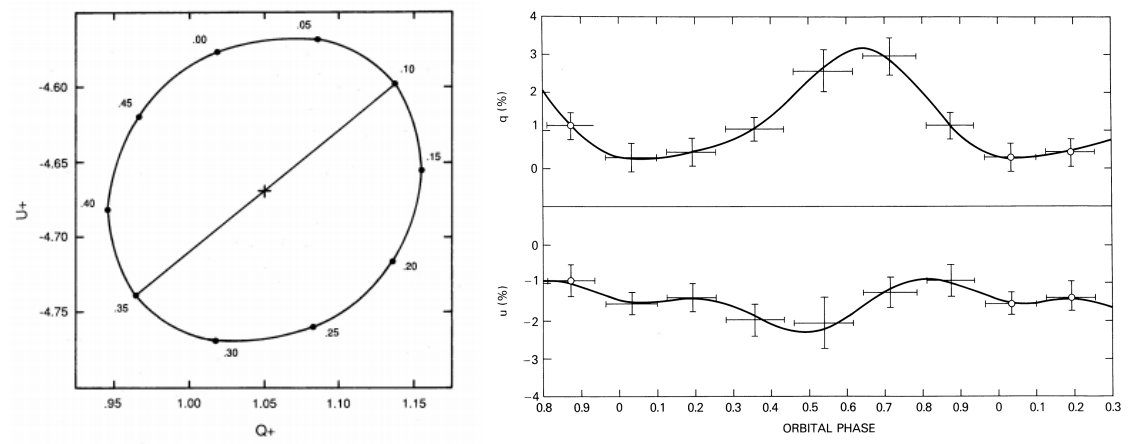

Fig. 16 Left: The observed Stokes optical linear polarization of Cyg X-1 in the $Q-U$ plane [143]. The values trace an ellipse, the eccentricity of which can be used to determine the inclination angle. Right: The observed $Q$ and $U$ time-resolved linear $V$-band polarimetric light curve of A0620-00 [134]. The solid line represents the second order Fourier fit to the data, which is then used to determine the inclination angle. (C)AAS. Reproduced with permission.

\section{References}

1. D.T. Wickramasinghe, L. Ferrario, PASP, 112, 873 (2000)

2. L. Ferrario, D. de Martino, B.T. Gänsicke, Space Sci. Rev., 191, 111 (2015)

3. M. Livio, J.E. Pringle, ApJ, 427, 956 (1994)

4. M. Cropper, Space Sci. Rev., 54, 195 (1990)

5. S.B. Potter, in Astronomy at High Angular Resolution, Astrophysics and Space Science Library, vol. 439, ed. by H.M.J. Boffin, G. Hussain, J.P. Berger, L. Schmidtobreick (2016), Astrophysics and Space Science Library, vol. 439, p. 179

6. A.J. Norton, MNRAS, 265, 316 (1993)

7. J. Patterson, PASP, 106, 209 (1994)

8. J. Patterson, ApJ, 234, 978 (1979). DOI 10.1086/157582

9. M. Eracleous, K. Horne, E.L. Robinson, E.H. Zhang, T.R. Marsh, J.H. Wood, ApJ, 433, 313 (1994). DOI 10.1086/174647

10. K. Reinsch, K. Beuermann, H. Hanusch, H.C. Thomas, in Magnetic Cataclysmic Variables, Astronomical Society of the Pacific Conference Series, vol. 85, ed. by D.A.H. Buckley, B. Warner (1995), Astronomical Society of the Pacific Conference Series, vol. 85, p. 115

11. C.S. Choi, T. Dotani, P.C. Agrawal, ApJ, 525, 399 (1999). DOI 10.1086/307873

12. W.F. Welsh, K. Horne, R. Gomer, MNRAS, 298, 285 (1998). DOI 10.1046/j.1365-8711. 1998.01643.x

13. G.A. Wynn, A.R. King, K. Horne, MNRAS, 286, 436 (1997). DOI 10.1093/mnras/286.2.436

14. N.R. Ikhsanov, A\&A, 338, 521 (1998)

15. N.R. Ikhsanov, ApJ, 640, L59 (2006). DOI 10.1086/503288

16. A.A. Blinova, M.M. Romanova, G.V. Ustyugova, A.V. Koldoba, R.V.E. Lovelace, arXiv eprints (2018)

17. G. Chanmugam, G.A. Dulk, ApJ, 244, 569 (1981)

18. S.M.A. Meggitt, D.T. Wickramasinghe, MNRAS, 198, 71 (1982)

19. D.T. Wickramasinghe, S.M.A. Meggitt, MNRAS, 214, 605 (1985)

20. L. Ferrario, D.T. Wickramasinghe, ApJ, 357, 582 (1990)

21. S.B. Potter, P.J. Hakala, M. Cropper, MNRAS, 297, 1261 (1998)

22. S.B. Potter, M. Cropper, P.J. Hakala, MNRAS, 315, 423 (2000)

23. T.R. Marsh, K. Horne, MNRAS, 235, 269 (1988) 
24. C.A. Watson, V.S. Dhillon, MNRAS, 326, 67 (2001)

25. S.B. Potter, E. Romero-Colmenero, C.A. Watson, D.A.H. Buckley, A. Phillips, MNRAS, 348, 316 (2004)

26. J.E.R. Costa, C.V. Rodrigues, MNRAS, 398, 240 (2009)

27. L. Ferrario, J. Bailey, D.T. Wickramasinghe, MNRAS, 262, 285 (1993)

28. D.T. Wickramasinghe, L. Ferrario, J. Bailey, ApJ, 342, L35 (1989)

29. L. Ferrario, D.T. Wickramasinghe, J. Bailey, I.R. Tuohy, J.H. Hough, ApJ, 337, 832 (1989)

30. D.T. Wickramasinghe, M. Cropper, K.O. Mason, M. Garlick, MNRAS, 250, 692 (1991)

31. M. Cropper, D.T. Wickramasinghe, MNRAS, 260, 696 (1993)

32. R.K. Campbell, T.E. Harrison, A.D. Schwope, S.B. Howell, ApJ, 672, 531 (2008)

33. V. Piirola, P. Hakala, G.V. Coyne, ApJ, 410, L107 (1993)

34. H. Vaeth, G. Chanmugam, J. Frank, ApJ, 457, 407 (1996)

35. S.B. Potter, M. Cropper, K.O. Mason, J.H. Hough, J.A. Bailey, MNRAS, 285, 82 (1997)

36. H. Vaeth, A\&A, 317, 476 (1997)

37. S. Katajainen, O.W. Butters, A.J. Norton, H.J. Lehto, V. Piirola, A\&A, 475, 1011 (2007)

38. V. Piirola, T. Vornanen, A. Berdyugin, G.V. Coyne, S. J., ApJ, 684, 558 (2008)

39. G. Chanmugam, A. Ray, ApJ, 285, 252 (1984)

40. J.R. Thorstensen, F.A. Ringwald, R.A. Wade, G.D. Schmidt, J.E. Norsworthy, AJ, 102, 272 (1991)

41. P. Rodríguez-Gil, B.T. Gänsicke, H.J. Hagen, S. Araujo-Betancor, A. Aungwerojwit, C. Allende Prieto, D. Boyd, J. Casares, D. Engels, O. Giannakis, E.T. Harlaftis, J. Kube, H. Lehto, I.G. Martínez-Pais, R. Schwarz, W. Skidmore, A. Staude, M.A.P. Torres, MNRAS, 377, 1747 (2007)

42. P. Rodríguez-Gil, J. Casares, I.G. Martínez-Pais, P. Hakala, D. Steeghs, ApJ, 548, L49 (2001)

43. I.G. Martínez-Pais, P. Rodríguez-Gil, J. Casares, MNRAS, 305, 661 (1999)

44. P. Rodríguez-Gil, J. Casares, I.G. Martínez-Pais, P.J. Hakala, in The Physics of Cataclysmic Variables and Related Objects, Astronomical Society of the Pacific Conference Series, vol. 261, ed. by B.T. Gänsicke, K. Beuermann, K. Reinsch (2002), Astronomical Society of the Pacific Conference Series, vol. 261, p. 533

45. P. Rodríguez-Gil, I.G. Martínez-Pais, J. de la Cruz Rodríguez, MNRAS, 395, 973 (2009)

46. D.S. Baskill, P.J. Wheatley, J.P. Osborne, MNRAS, 357, 626 (2005)

47. T.R. Marsh, B.T. Gänsicke, S. Hümmerich, F.J. Hambsch, K. Bernhard, C. Lloyd, E. Breedt, E.R. Stanway, D.T. Steeghs, S.G. Parsons, O. Toloza, M.R. Schreiber, P.G. Jonker, J. van Roestel, T. Kupfer, A.F. Pala, V.S. Dhillon, L.K. Hardy, S.P. Littlefair, A. Aungwerojwit, S. Arjyotha, D. Koester, J.J. Bochinski, C.A. Haswell, P. Frank, P.J. Wheatley, Nature, 537, 374 (2016). DOI 10.1038/nature18620

48. J.I. Katz, ApJ, 835, 150 (2017). DOI 10.3847/1538-4357/835/2/150

49. D.A.H. Buckley, P.J. Meintjes, S.B. Potter, T.R. Marsh, B.T. Gänsicke, Nature Astronomy 1, 0029 (2017). DOI 10.1038/s41550-016-0029

50. R. Fender, E. Gallo, Space Sci. Rev., 183, 323 (2014)

51. J.E. McClintock, R.A. Remillard, Black hole binaries (Cambridge University Press, 2006), pp. 157-213

52. T.M. Belloni, in Lecture Notes in Physics, Berlin Springer Verlag, Lecture Notes in Physics, Berlin Springer Verlag, vol. 794, ed. by T. Belloni (2010), Lecture Notes in Physics, Berlin Springer Verlag, vol. 794, p. 53

53. K.I.I. Koljonen, D.M. Russell, J.M. Corral-Santana, M. Armas Padilla, T. Muñoz-Darias, F. Lewis, M. Coriat, F.E. Bauer, MNRAS, 460, 942 (2016)

54. R. Narayan, I. Yi, ApJ, 428, L13 (1994)

55. R.P. Fender, MNRAS, 322, 31 (2001)

56. E. Gallo, R.P. Fender, G.G. Pooley, MNRAS, 344, 60 (2003)

57. D.M. Russell, J.C.A. Miller-Jones, T.J. Maccarone, Y.J. Yang, R.P. Fender, F. Lewis, ApJ, 739, L19 (2011)

58. D.L. Meier, ApJ, 548, L9 (2001)

59. R.D. Blandford, A. Königl, ApJ, 232, 34 (1979) 
60. P. Polko, D.L. Meier, S. Markoff, ApJ, 723, 1343 (2010)

61. M. Coriat, Jets relativistes des trous noirs accrtants. Ph.D. thesis, Paris (2010)

62. R.P. Fender, G.G. Pooley, P. Durouchoux, R.P.J. Tilanus, C. Brocksopp, MNRAS, 312, 853 (2000)

63. S. Markoff, H. Falcke, R. Fender, A\&A, 372, L25 (2001)

64. C.R. Kaiser, MNRAS, 367, 1083 (2006)

65. J. Malzac, ArXiv e-prints (2018)

66. C.R. Kaiser, MNRAS, 360, 176 (2005)

67. S. Corbel, R.P. Fender, ApJ, 573, L35 (2002)

68. D.M. Russell, R.P. Fender, R.I. Hynes, C. Brocksopp, J. Homan, P.G. Jonker, M.M. Buxton, MNRAS, 371, 1334 (2006)

69. D. Maitra, S. Markoff, C. Brocksopp, M. Noble, M. Nowak, J. Wilms, MNRAS, 398, 1638 (2009)

70. P. Gandhi, A.W. Blain, D.M. Russell, P. Casella, J. Malzac, S. Corbel, P. D'Avanzo, F.W. Lewis, S. Markoff, M. Cadolle Bel, P. Goldoni, S. Wachter, D. Khangulyan, A. Mainzer, ApJ, 740, L13 (2011)

71. D.M. Russell, T.D. Russell, J.C.A. Miller-Jones, K. O’Brien, R. Soria, G.R. Sivakoff, T. Slaven-Blair, F. Lewis, S. Markoff, J. Homan, D. Altamirano, P.A. Curran, M.P. Rupen, T.M. Belloni, M. Cadolle Bel, P. Casella, S. Corbel, V. Dhawan, R.P. Fender, E. Gallo, P. Gandhi, S. Heinz, E.G. Körding, H.A. Krimm, D. Maitra, S. Migliari, R.A. Remillard, C.L. Sarazin, T. Shahbaz, V. Tudose, ApJ, 768, L35 (2013)

72. S. Corbel, H. Aussel, J.W. Broderick, P. Chanial, M. Coriat, A.J. Maury, M.M. Buxton, J.A. Tomsick, A.K. Tzioumis, S. Markoff, J. Rodriguez, C.D. Bailyn, C. Brocksopp, R.P. Fender, P.O. Petrucci, M. Cadolle-Bel, D. Calvelo, L. Harvey-Smith, MNRAS, 431, L107 (2013)

73. R.I. Hynes, E.L. Robinson, K.J. Pearson, D.M. Gelino, W. Cui, Y.Q. Xue, M.A. Wood, T.K. Watson, D.E. Winget, I.M. Silver, ApJ, 651, 401 (2006)

74. S. Chaty, G. Dubus, A. Raichoor, A\&A, 529, A3 (2011)

75. D.M. Russell, S. Markoff, P. Casella, A.G. Cantrell, R. Chatterjee, R.P. Fender, E. Gallo, P. Gandhi, J. Homan, D. Maitra, J.C.A. Miller-Jones, K. O’Brien, T. Shahbaz, MNRAS, 429, $815(2013)$

76. A.J. Tetarenko, G.R. Sivakoff, J.C.A. Miller-Jones, M. Bremer, K.P. Mooley, R.P. Fender, C. Rumsey, A. Bahramian, D. Altamirano, S. Heinz, D. Maitra, S.B. Markoff, S. Migliari, M.P. Rupen, D.M. Russell, T.D. Russell, C.L. Sarazin, MNRAS, 482, 2950 (2019)

77. T.D. Russell, R. Soria, J.C.A. Miller-Jones, P.A. Curran, S. Markoff, D.M. Russell, G.R. Sivakoff, MNRAS, 439, 1390 (2014)

78. F. Rahoui, J.C. Lee, S. Heinz, D.C. Hines, K. Pottschmidt, J. Wilms, V. Grinberg, ApJ, 736, 63 (2011)

79. R.M. Plotkin, E. Gallo, P.G. Jonker, J.C.A. Miller-Jones, J. Homan, T. Muñoz-Darias, S. Markoff, M. Armas Padilla, R. Fender, A.P. Rushton, D.M. Russell, M.A.P. Torres, MNRAS, 456, 2707 (2016)

80. T. Dinçer, C.D. Bailyn, J.C.A. Miller-Jones, M. Buxton, R.K.D. MacDonald, ApJ, 852, 4 (2018)

81. D.M. Russell, A.A. Qasim, F. Bernardini, R.M. Plotkin, F. Lewis, K.I.I. Koljonen, Y.J. Yang, ApJ, 852, 90 (2018)

82. S. Migliari, J.A. Tomsick, J.C.A. Miller-Jones, S. Heinz, R.I. Hynes, R.P. Fender, E. Gallo, P.G. Jonker, T.J. Maccarone, ApJ, 710, 117 (2010)

83. M. Díaz Trigo, S. Migliari, J.C.A. Miller-Jones, F. Rahoui, D.M. Russell, V. Tudor, A\&A, 600, A8 (2017)

84. M. Díaz Trigo, D. Altamirano, T. Dinçer, J.C.A. Miller-Jones, D.M. Russell, A. Sanna, C. Bailyn, F. Lewis, S. Migliari, F. Rahoui, A\&A, 616, A23 (2018)

85. K.I.I. Koljonen, D.M. Russell, J.A. Fernández-Ontiveros, S. Markoff, T.D. Russell, J.C.A. Miller-Jones, A.J. van der Horst, F. Bernardini, P. Casella, P.A. Curran, P. Gandhi, R. Soria, ApJ, 814, 139 (2015)

86. C. Ceccobello, Y. Cavecchi, M.H.M. Heemskerk, S. Markoff, P. Polko, D. Meier, MNRAS, 473, 4417 (2018) 
87. M. Durant, P. Gandhi, T. Shahbaz, A.P. Fabian, J. Miller, V.S. Dhillon, T.R. Marsh, ApJ, 682, L45 (2008)

88. M. Durant, T. Shahbaz, P. Gandhi, R. Cornelisse, T. Muñoz-Darias, J. Casares, V. Dhillon, T. Marsh, H. Spruit, K. O’Brien, D. Steeghs, R. Hynes, MNRAS, 410, 2329 (2011)

89. P. Gandhi, K. Makishima, M. Durant, A.C. Fabian, V.S. Dhillon, T.R. Marsh, J.M. Miller, T. Shahbaz, H.C. Spruit, MNRAS, 390, L29 (2008)

90. P. Gandhi, V.S. Dhillon, M. Durant, A.C. Fabian, A. Kubota, K. Makishima, J. Malzac, T.R. Marsh, J.M. Miller, T. Shahbaz, H.C. Spruit, P. Casella, MNRAS, 407, 2166 (2010)

91. R.I. Hynes, C.A. Haswell, W. Cui, C.R. Shrader, K. O'Brien, S. Chaty, D.R. Skillman, J. Patterson, K. Horne, MNRAS, 345, 292 (2003)

92. P. Casella, T.J. Maccarone, K. O’Brien, R.P. Fender, D.M. Russell, M. van der Klis, A. Pe'Er, D. Maitra, D. Altamirano, T. Belloni, G. Kanbach, M. Klein-Wolt, E. Mason, P. Soleri, A. Stefanescu, K. Wiersema, R. Wijnands, MNRAS, 404, L21 (2010)

93. A. Veledina, J. Poutanen, I. Vurm, ApJ, 737, L17 (2011)

94. T. Shahbaz, R.P. Fender, C.A. Watson, K. O’Brien, ApJ, 672, 510 (2008)

95. K. Serkowski, in Interstellar Dust and Related Topics, IAU Symposium, vol. 52, ed. by J.M. Greenberg, H.C. van de Hulst (1973), IAU Symposium, vol. 52, p. 145

96. J. Schultz, P. Hakala, J. Huovelin, Baltic Astronomy 13, 581 (2004)

97. R. Blandford, E. Agol, A. Broderick, J. Heyl, L. Koopmans, H.W. Lee, in Astrophysical Spectropolarimetry, ed. by J. Trujillo-Bueno, F. Moreno-Insertis, F. Sánchez (2002), pp. 177223

98. D.M. Russell, T. Shahbaz, MNRAS, 438, 2083 (2014)

99. V.L. Ginzburg, S.I. Syrovatskii, ARA\&A, 3, 297 (1965)

100. K.H. Nordsieck, ApJ, 209, 653 (1976)

101. M.S. Longair, High Energy Astrophysics (Cambridge University Press, 2011)

102. G.B. Rybicki, A.P. Lightman, Radiative Processes in Astrophysics (Wiley-Interscience, 1986)

103. C.I. Bjornsson, G.R. Blumenthal, ApJ, 259, 805 (1982)

104. C.I. Bjornsson, MNRAS, 216, 241 (1985)

105. A.G. Pacholczyk, Radio astrophysics. Nonthermal processes in galactic and extragalactic sources (Freeman, 1970)

106. L. Rudnick, F.N. Owen, T.W. Jones, J.J. Puschell, W.A. Stein, ApJ, 225, L5 (1978)

107. G. Dubus, S. Chaty, A\&A, 458, 591 (2006)

108. D.M. Russell, R.P. Fender, MNRAS, 387, 713 (2008)

109. D.M. Russell, P. Casella, R. Fender, P. Soleri, M.L. Pretorius, F. Lewis, M. van der Klis, ArXiv e-prints (2011)

110. M.C. Baglio, D. Mainetti, P. D’Avanzo, S. Campana, S. Covino, D.M. Russell, T. Shahbaz, A\&A, 572, A99 (2014)

111. T. Shahbaz, D.M. Russell, S. Covino, K. Mooley, R.P. Fender, C. Rumsey, MNRAS, 463, $1822(2016)$

112. V.M. Lipunov, E. Gorbovskoy, V. Kornilov, V. Krushinskiy, D. Vlasenko, N. Tiurina, P. Balanutsa, A. Kuznetsov, N. Budnev, O. Gress, A. Tlatov, R. Rebolo Lopez, M. Serra-Ricart, D.A.H. Buckley, G. Israelyan, N. Lodieu, K. Ivanov, S. Yazev, Y. Sergienko, A. Gabovich, V. Yurkov, H. Levato, C. Saffe, R. Podesta, C. Mallamaci, C. Lopez, ApJ, 833, 198 (2016)

113. J. Rodriguez, M. Cadolle Bel, J. Alfonso-Garzón, T. Siegert, X.L. Zhang, V. Grinberg, V. Savchenko, J.A. Tomsick, J. Chenevez, M. Clavel, S. Corbel, R. Diehl, A. Domingo, C. Gouiffès, J. Greiner, M.G.H. Krause, P. Laurent, A. Loh, S. Markoff, J.M. Mas-Hesse, J.C.A. Miller-Jones, D.M. Russell, J. Wilms, A\&A, 581, L9 (2015)

114. M.T. Reynolds, R.C. Reis, J.M. Miller, E.M. Cackett, N. Degenaar, MNRAS, 441, 3656 (2014)

115. E. Gallo, S. Migliari, S. Markoff, J.A. Tomsick, C.D. Bailyn, S. Berta, R. Fender, J.C.A. Miller-Jones, ApJ, 670, 600 (2007)

116. R.M. Plotkin, E. Gallo, S. Markoff, J. Homan, P.G. Jonker, J.C.A. Miller-Jones, D.M. Russell, S. Drappeau, MNRAS, 446, 4098 (2015) 
117. E. Gallo, R.P. Fender, R.I. Hynes, MNRAS, 356, 1017 (2005)

118. E. Gallo, R.P. Fender, J.C.A. Miller-Jones, A. Merloni, P.G. Jonker, S. Heinz, T.J. Maccarone, M. van der Klis, MNRAS, 370, 1351 (2006)

119. E. Gallo, J.C.A. Miller-Jones, D.M. Russell, P.G. Jonker, J. Homan, R.M. Plotkin, S. Markoff, B.P. Miller, S. Corbel, R.P. Fender, MNRAS, 445, 290 (2014)

120. J.C.A. Miller-Jones, P.G. Jonker, T.J. Maccarone, G. Nelemans, D.E. Calvelo, ApJ, 739, L18 (2011)

121. J.C.A. Miller-Jones, J. Strader, C.O. Heinke, T.J. Maccarone, M. van den Berg, C. Knigge, L. Chomiuk, E. Noyola, T.D. Russell, A.C. Seth, G.R. Sivakoff, MNRAS, 453, 3918 (2015)

122. L. Chomiuk, J. Strader, T.J. Maccarone, J.C.A. Miller-Jones, C. Heinke, E. Noyola, A.C. Seth, S. Ransom, ApJ, 777, 69 (2013)

123. S.A. Dzib, M. Massi, F. Jaron, A\&A, 580, L6 (2015)

124. B.E. Tetarenko, A. Bahramian, R.M. Arnason, J.C.A. Miller-Jones, S. Repetto, C.O. Heinke, T.J. Maccarone, L. Chomiuk, G.R. Sivakoff, J. Strader, F. Kirsten, W. Vlemmings, ApJ, 825, 10 (2016)

125. V. Rana, A. Loh, S. Corbel, J.A. Tomsick, D. Chakrabarty, D.J. Walton, D. Barret, S.E. Boggs, F.E. Christensen, W. Craig, F. Fuerst, P. Gandhi, B.W. Grefenstette, C. Hailey, F.A. Harrison, K.K. Madsen, F. Rahoui, D. Stern, S. Tendulkar, W.W. Zhang, ApJ, 821, 103 (2016)

126. T. Shahbaz, D.M. Russell, C. Zurita, J. Casares, J.M. Corral-Santana, V.S. Dhillon, T.R. Marsh, MNRAS, 434, 2696 (2013)

127. S. Markoff, M.A. Nowak, E. Gallo, R. Hynes, J. Wilms, R.M. Plotkin, D. Maitra, C.V. Silva, S. Drappeau, ApJ, 812, L25 (2015)

128. D.M. Gelino, C.R. Gelino, T.E. Harrison, ApJ, 718, 1 (2010)

129. C.S. Froning, A.G. Cantrell, T.J. Maccarone, K. France, J. Khargharia, L.M. Winter, E.L. Robinson, R.I. Hynes, J.W. Broderick, S. Markoff, M.A.P. Torres, M. Garcia, C.D. Bailyn, J.X. Prochaska, J. Werk, C. Thom, S. Béland, C.W. Danforth, B. Keeney, J.C. Green, ApJ, 743, 26 (2011)

130. M.C. Baglio, P. D’Avanzo, T. Muñoz-Darias, R.P. Breton, S. Campana, A\&A, 559, A42 (2013)

131. D.M. Russell, T. Shahbaz, F. Lewis, E. Gallo, MNRAS, 463, 2680 (2016)

132. E. Kuulkers, R.P. Fender, R.E. Spencer, R.J. Davis, I. Morison, MNRAS, 306, 919 (1999)

133. J.C. Brown, I.S. McLean, A.G. Emslie, A\&A, 68, 415 (1978)

134. J.F. Dolan, S. Tapia, PASP, 101, 1135 (1989)

135. L. Drissen, R. Lamontagne, A.F.J. Moffat, P. Bastien, M. Seguin, ApJ, 304, 188 (1986)

136. R.J. Rudy, J.C. Kemp, ApJ, 221, 200 (1978)

137. J.C. Brown, C. Aspin, J.F.L. Simmons, I.S. McLean, MNRAS, 198, 787 (1982)

138. J.F.L. Simmons, C.B. Boyle, A\&A, 134, 368 (1984)

139. C. Aspin, J.F.L. Simmons, J.C. Brown, MNRAS, 194, 283 (1981)

140. J.F.L. Simmons, C. Aspin, J.C. Brown, MNRAS, 198, 45 (1982)

141. K.G. Wolinski, J.F. Dolan, MNRAS, 267, 5 (1994)

142. J.C. Kemp, M.S. Barbour, T.E. Parker, L.C. Herman, ApJ, 228, L23 (1979)

143. J.F. Dolan, S. Tapia, ApJ, 344, 830 (1989)

144. O. Nagae, K.S. Kawabata, Y. Fukazawa, A. Okazaki, M. Isogai, T. Yamashita, AJ, 137, 3509 (2009) 